\title{
A complex associated with a symmetric matrix
}

\author{
By \\ Shiro Goto and Sadao TACHIBANA \\ (Communicated by Prof. Nagata March 15, 1976)
}

\section{Introduction.}

In this paper let $R$ be a commutative ring in which 2 is invertible and let $X$ be a symmetric $n \times n$ matrix with entries in $R(n \geqq 2)$. We denote by a the ideal of $R$ generated by all the $(n-1) \times(n-1)$ minors of $X$. It is known that depth $\mathfrak{a} \leqq 3$ if $R$ is Noetherian (c. f. Theorem 1, [4]). (Here depth a denotes the common length of maximal $R$-sequences contained in a.)

The purpose of this note is to construct a complex $C^{x}$ associated with $X$ of finitely generated free $R$-modules of length 3 and to show that $C^{X}$ provides the resolution of $R / \mathfrak{a}$ if $R$ is Noetherian and if depth $a=3$.

T. H. Gulliksen and O. G. Negäd [3] constructed an elegant complex $\tilde{C}^{x}$ associated with $X$ but this complex is too big to provide the resolution of $R / a$ in the present case. Nevertheless we will find that $C^{X}$ is a direct summand of $\tilde{C}^{x}$ as a complex and this is available to study some properties of $C^{x}$.

\section{Construction of $\boldsymbol{C}^{x}$.}

In the following let $M_{n}(R)$ denote the free $R$-module of all the $n \times n$ matrices with entries in $R$ and we put

$$
\begin{aligned}
& A=\left\{S \in M_{n}(R) /{ }^{t} S=S\right\}, \\
& B=\left\{T \in M_{n}(R) /{ }^{t} T=-T\right\}, \\
& C=\left\{M \in M_{n}(R) / \text { Trace } M=0\right\} .
\end{aligned}
$$

Note that $A, B$ and $C$ are finitely generated free $R$-modules of $\operatorname{rank}\left(\begin{array}{c}n+1 \\ 2\end{array}\right),\left(\begin{array}{c}n \\ 2\end{array}\right)$ and $n^{2}-1$ respectively. We denote by $Y$ the matrix of cofactors of $X$.

Construction of $C^{x}$.

We put $C_{3}{ }^{x}=B, C_{2}{ }^{x}=C, C_{1}{ }^{x}=A, C_{0}{ }^{x}=R$, and $C_{i}{ }^{x}=(0)$ if $i<0$ or $i>3$. For the differential $d$ we define

$$
\begin{aligned}
& d_{3}(T)=X T \text { for } T \in B, \\
& d_{2}(M)=M X+{ }^{t}(M X) \text { for } M \in C, \\
& d_{1}(S)=\text { Trace } Y S \text { for } S \in A .
\end{aligned}
$$

It is easy to check that $C^{x}$ is actually a complex with $H_{0}\left(C^{x}\right)=R / \mathfrak{a}$.

First we note

Lemma 1. Suppose that $f: R \rightarrow R^{\prime}$ is a homomorphism of rings and let $X^{\prime}$ denote the matrix obtained by applying $f$ to the entries of $X$. Then 
$C^{X^{\prime}} \cong C^{x} \otimes_{R} R^{\prime}$

\section{Relation between $\boldsymbol{C}^{x}$ and $\tilde{\boldsymbol{C}}^{x}$.}

We recall the construction of $\tilde{C}^{x}([3]): \tilde{C}_{4}{ }^{x}=\tilde{C}_{0}{ }^{x}=R, \tilde{C}_{3}{ }^{x}=\tilde{C}_{1}{ }^{x}=M_{n}(R)$, and $\tilde{C}_{2}{ }^{x}$ is given by the homology of the complex $R \stackrel{i}{\rightarrow} M_{n}(R) \oplus M_{n}(R) \stackrel{j}{\rightarrow} R$ where $i(r)=(r I, r I)$ for $r \in R$ and $j(M, N)=$ Trace $(M-N)$ for $M, N \in M_{n}(R)$. (Here $I$ denotes the $n \times n$ unit matrix.) The differential $d$ is defined as follows:

$d_{4}(r)=r Y$ for $r \in R$,

$d_{3}(M)=$ the class of $(X M, M X)$ for $M \in M_{n}(R)$,

$d_{2}$ (the class of $\left.(M, N)\right)=M X-X N$ for $M, N \in M_{n}(R)$ with Trace $M=$ Trace $N$,

$d_{1}(M)=\operatorname{Trace} Y M$ for $M \in M_{n}(R)$.

The following is the key lemma of this paper:

Lemma 2. $C^{x}$ is a direct summand of $\tilde{C}^{x}$ as a complex.

Proof. If we define the $R$-linear maps $p: M_{n}(R) \rightarrow B, q: \tilde{C}_{2}{ }^{x} \rightarrow C$, $r: M_{n}(R) \rightarrow A$ and $s: C \rightarrow \tilde{C}_{2}{ }^{x}$ by

$p(M)=\left(M-{ }^{t} M\right) / 2$ for $M \in M_{n}(R)$,

$q$ (the class of $(M, N))=\left(M-{ }^{t} N\right) / 2$ for $M, N \in M_{n}(R)$ with Trace $M=$ $\operatorname{Trace} N$,

$r(M)=\left(M+{ }^{t} M\right) / 2$ for $M \in M_{n}(R)$,

$s(M)=$ the class of $\left(M,-{ }^{t} M\right)$ for $M \in C$,

then some straightforward calculation shows that the following diagram is commutative:

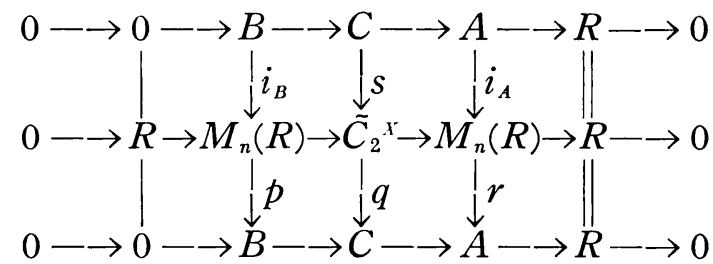

where $i_{B}: B \rightarrow M_{n}(R)$ and $i_{A}: A \rightarrow M_{n}(R)$ denote the inclusion maps. Of course $p \circ i_{B}=1_{B}, q \circ S=1_{C}$ and $r \circ i_{A}=1_{A}$.

Corollary. Suppose that $E$ is an arbitrary R-module. Then $\mathfrak{a}^{2} H\left(C^{x} \otimes_{R} E\right)=(0)$.

Proof. $H\left(C^{x} \otimes_{R} E\right)$ is an $R$-submodule of $H\left(\tilde{C^{x}} \otimes_{R} E\right)$ by Lemma 2 and hence the assertion is obvious since $a^{2} H\left(\tilde{C}^{x} \otimes_{R} E\right)=(0)$ (c.f. Lemma 2 and 4 , [3]).

Corllary. Let $E$ be an $R$-module and suppose that $\operatorname{Supp}_{R} E \cap \operatorname{Spec} R / a$ $=\phi$. Then we have $H\left(C^{x} \otimes_{R} E\right)=(0)$. 


\section{Main results.}

Theorem. Suppose that $R$ is Noetherian and let $E$ be a finitely generated $R$-module such that $E \neq a E$. Then we have

$$
\operatorname{depth}(\mathfrak{a}, E)+\max \left\{i \in \boldsymbol{Z} / H_{i}\left(\boldsymbol{C}^{x} \underset{R}{\otimes} E\right) \neq(0)\right\}=3
$$

where depth( $(a, E)$ denotes the common length of maximal E-sequences contained in a.

Proof. We will prove by induction on $t=\operatorname{depth}(\mathfrak{a}, E)$.

$(t=0)$ We must show that $H_{3}\left(C^{x} \otimes_{R} E\right) \neq(0)$. By the assumption, a $e=(0)$ for some $0 \neq e \in E$. Since $H_{3}\left(C^{x} \otimes_{R} E\right)$ contains $H_{3}\left(C^{x} \otimes_{R} R e\right)$ as an $R$-submodule, we may assume that $E=R e$. Moreover, because $C^{x} \otimes_{R} E \cong\left(C^{x} \otimes_{R} \bar{R}\right) \otimes_{\bar{R}} E$ where $R=\bar{R} /(0): E$, we may assume further that $(0): E=(0)$. Thus it suffices to prove in case $E=R$ and $\mathfrak{a}=(0)$. Now suppose that $H_{3}\left(C^{X}\right)=(0)$. Then $H_{3}\left(C^{x} \otimes_{R} R_{\mathfrak{p}}\right)=(0)$ for every $\mathfrak{p} \in \operatorname{Spec} R$ and so, to obtain a contradiction, we can assume that $(R, \mathfrak{m})$ is a local ring of $\operatorname{depth} R=0$ after localization at $\mathfrak{p}$ for some $\mathfrak{p} \in \operatorname{Ass} R$. In this situation, $M=$ Coker $d_{3}$ is a free $R$-module since $\mathrm{hd}_{R} M \leqq 1$ and consequently $d_{3}$ is a split monomorphism. Thus, applying $\bigotimes_{R} R / \mathrm{m}$, we can assume that $R$ is a field and so we have ${ }^{t} P X P=\left(\begin{array}{cc}D & 0 \\ 0 & 0\end{array}\right)$ for some invertible $n \times n$ matrix $P$ where $D$ is an $s \times s$ diagonal matrix $(s=\operatorname{rank} X)$. Now consider an $n \times n$ matrix $T=\left(\begin{array}{rrr}0 & 0 & \\ 0 & 0 & 1 \\ -1 & 0\end{array}\right)$. Then $T \in B$ and $\left({ }^{t} P X P\right) T=0$ since $s \leqq n-2$. (Recall that $\mathfrak{a}=(0)$.) Therefore $X\left(P T^{t} P\right)=0$ with $0 \neq P T^{t} P \in B$ - this is the required contradiction.

$(t>0)$ Let $f$ be an $E$-regular element contained in $\mathfrak{a}^{2}$. Applying $C^{x} \otimes_{R}$ to the exact sequence $0 \rightarrow E \stackrel{f}{\rightarrow} E \rightarrow E / f E \rightarrow 0$, we have an exact sequence

$$
0 \rightarrow C^{x} \underset{R}{\otimes} E \stackrel{f}{\rightarrow} C^{x} \underset{R}{\bigotimes_{R}} E \rightarrow C^{x} \underset{R}{\bigotimes_{R}} E / f E \rightarrow 0
$$

of complexes. This yields an exact sequence of homologies

$$
0 \rightarrow H_{i}\left(C^{x} \underset{R}{\otimes} E\right) \rightarrow H_{i}\left(C^{x} \underset{R}{\otimes} E / f E\right) \rightarrow H_{i-1}\left(C^{x} \bigotimes_{R} E\right) \rightarrow 0
$$

for every $i \in \boldsymbol{Z}$ by the corollary of Lemma 2 , since $f \in \mathfrak{a}^{2}$. By the hypothesis of induction, we have known that $(t-1)+\max \left\{i \in \boldsymbol{Z} / H_{i}\left(C^{x} \otimes_{R} E / f E\right) \neq(0)\right\}=3$ and so the result follows from $(*)$.

Corollary. Suppose that $R$ is Noetherian and assume that depth $a=3$. Then $C^{X}$ provides a resolution of $R / \mathrm{a}$.

For a Macaulay local ring $(R, \mathfrak{m})$ we put $r(R)=\operatorname{dim} R / \mathfrak{m} \operatorname{Ext}_{R}^{S}(R / \mathfrak{m}, R)(s=$ $\operatorname{dim} R$ ) and call it the type of $R$. It is known that $R$ is a Gorenstein ring if and only if $r(R)=1$ (c.f. [2]).

Corollary. Let $k$ be a field of $\operatorname{ch} k \neq 2$ and let $R=k\left[\left\{X_{i j}\right\}_{1 \leqq i \leq j \leq n}\right]$ be 
a polynomial ring. We put $X=\left(X_{i j}\right)_{1 \leqq i, j \leqq n}\left(X_{i j}=X_{j i}\right.$ if $\left.i>j\right)$. Then $C^{x}$ provides a resolution of $R / \mathfrak{a}$ with $r\left(R_{\mathfrak{m}} / a R_{\mathfrak{m}}\right)=\left(\begin{array}{l}n \\ 2\end{array}\right)$ where $\mathfrak{m}$ denotes the maximal ideal $\left(\left\{X_{i j}\right\}_{1 \leqq i \leqq j \leqslant n}\right)$ of $R$. In particular $R / \mathfrak{a}$ is a Gorenstein ring if and only if $n=2$.

Proof. By [4], we know that $R / \mathfrak{a}$ is a Macaulay ring of depth $a=3$. Thus $C^{x} \otimes_{R} R_{\mathfrak{m}}$ is a minimal free resolution of $R_{\mathfrak{m}} / \mathfrak{a} R_{\mathfrak{m}}$ in this case and consequently we know that $r\left(R_{\mathfrak{m}} / \mathfrak{a} R_{\mathfrak{m}}\right)=\left(\begin{array}{c}\mathrm{n} \\ 2\end{array}\right)$ (c. f. Lemma 3.5, [1]). Therefore $R_{\mathfrak{m}} / a R_{\mathfrak{m}}$ is a Gorenstein ring if and only if $n=2$ and it is known that $R / \mathfrak{a}$ is a Gorenstein ring globally if $R_{\mathrm{m}} / \mathrm{a} R_{\mathrm{m}}$ is a Gorenstein local ring (c.f. Theorem, [1]).

\section{DEPARTMENT OF MATHEMATICS NIHON UNIVERSITY}

\section{References}

[1] Y. Aoyama and S. Goto, On the type of graded Cohen-Macaulay rings, J. Kyoto Univ., 15 (1975), 19-23.

[2] H. Bass, On the ubiquity of Gorenstein rings, Math. Zeit., 82 (1963), 8-28.

[3] T. H. Gulliksen and O. G. Negärd, Un complexe résolvant pour certains idéaux déterminantiels, C. R. A.S., 274 (1972), 16-18.

[4] R. E. Kutz, Cohen-Macaulay rings and ideal theory in rings of invariants of algebraic groups, Trans. A. M. S., 194 (1974), 115-129. 